\title{
Frontiers of fractals for complex systems: recent advances and future challenges
}

\author{
A. Gowrisankar ${ }^{1, a}$ and Santo Banerjee ${ }^{2, b}$ \\ 1 Department of Mathematics, School of Advanced Sciences, Vellore Institute of Technology, Vellore, Tamil Nadu 632014 , \\ India \\ 2 Dipartimento di Scienze Matematiche, Politecnico di Torino, Corso Duca degli Abruzzi, 24, 10129 Turin, Italy
}

Published online 22 December 2021

(C) The Author(s), under exclusive licence to EDP Sciences, Springer-Verlag GmbH Germany, part of Springer Nature 2021

\begin{abstract}
This special issue of the European Physical Journal Special Topics titled "Frontiers of Fractals for Complex Systems: Recent Advances and Future Challenges" is a collection of cutting-edge research proposing the application of fractal features to the dynamics of highly nonlinear complex systems.
\end{abstract}

The fractal theory seeks to understand complexity and provide an innovative way to recognize irregularity and complex systems. Fractals allow us to see a certain symmetry and order even in otherwise seemingly disordered and complex systems. The importance of the discovery of fractals can hardly be overstated. Since their discovery there has been a surge of research activities geared towards using this powerful concept in almost every scientific discipline to gain deeper insights into many unsolved problems. A host of applications dealing with the fractal geometry of such diverse topics as price changes and salary distributions, turbulence, statistics of error in telephone messages, word frequencies in written texts, and aggregation and fragmentation processes are just a few examples.

Another reason for the development of fractals: advances in computer graphics. The computer has aided the researchers by providing once-unimaginable, fascinating fractal structures like the Mandelbrot set and Julia set. In mathematical literature, the Cantor set, Sierpinski triangle and von Koch curve are some of the most widely discussed fractal structures. For more detailed information on fractals, see [1-3].

In recent years, the role of fractal functions in the realm of approximation theory has become more prominent. Fractal interpolation functions (FIFs) have been introduced as the attractor of special type of the iterated function system (IFS). In contrast to classical methods, fractal interpolation functions produce complex or naturally occurring structures using a simple recursive procedure; as such, the fractal approach offers more versatility in approximation theory. In the development of FIF theory, in order to extend the potential utility of FIFs and enhance flexibility in the approxima-

\footnotetext{
a e-mail: gowrisankargri@gmail.com

b e-mail: santoban@gmail.com (corresponding author)
}

tion, many researchers have constructed various types of FIFs (see [1-7]).

The most fundamental characteristic of fractals is their dimension, which is not always an integer. The fractal dimension is estimated to understand the behavior of complex physical systems. Several numerical techniques have been developed to compute the fractal dimension. Definitions like Hausdorff and box counting have been suggested for the calculation of the fractal dimension in the field of fractal analysis. Though fractal analysis offers many salient features for describing complex systems, it has been found limiting in terms of describing objects that exhibit multifractal behavior. As a result, multifractals have arisen as a more complex form of fractals. The striking beauty of multifractal analysis is that it assigns a set of fractal dimensions (a spectrum) to a given object, whereas fractal analysis assigns a single fractal value. A variety of multifractal algorithms have been proposed for use in practical applications. Multifractal analysis, as an interesting tool, has also been used to characterize signals in medicine and the biological sciences (see [8-15]).

The advent in recent years of inexpensive computing power and graphics has led to the study of nontraditional geometric objects in many fields of science and engineering, while at the same time, fractals have been used to depict societal issues that can only be adequately described in terms of complex systems and the idea of fractals has been used to describe them. In a sense, fractal theory has brought many seemingly unrelated subjects under one umbrella. In recent years, the study of fractals has faced major changes and challenges with rapid technological advances, and many new factors now have to be considered.

This special issue is a collection of 21 cutting-edge research articles that address the application of fractal features to the dynamics of highly nonlinear complex systems. 
The articles can be divided into five sections, namely,

- Fractal functions

- Multifractality and applications

- Fractal calculus

- Chaotic systems

- Fractal dimension and applications

Section 1 includes an approximation of the continuous function defined on a subset of $\mathbb{R}^{2}$ with a nonself-referential bivariate fractal interpolation function. For smoother approximation of the prescribed function, an $r$-times continuously differentiable hidden variable bivariate FIF is developed [16].

Classical spherical harmonics are generalized with the construction of a set of square integrable functions in [17]. This generalization is made using the $\alpha$-fractal functions. In addition, the Bessel sequence, frame sequence and Riesz sequence for the square integrable functions are investigated.

A recurrent iterated function system is used to construct an affine recurrent FIF. Further, its convergence analysis and shape-preserving properties are studied in detail [18].

In [19], by defining a continuous function on the Sierpinski gasket, its associated $\alpha$-fractal function and its box dimension are investigated. Some properties like topological automorphism, boundedness, and Fredholm are discussed by defining the fractal operator on the gasket. In addition, it is proved that the fractal Schauder basis exists for the Sierpinski gasket.

In [20], the Riemann-Liouville fractional calculus is applied to the fractal interpolation function. The study distinguishes between the roles of constant scaling factors and function scaling factors in approximation. After introducing the fractional operator on the space of continuous functions defined on some closed interval of $\mathbb{R}$, a number of analytical and algebraic properties are discussed.

In [21], the Katugampola fractional integral of a vector valued function is discussed and it is shown that the fractional integral preserves the properties (like continuity and boundedness) of the original function. For the graph of the Katugampola fractional integral of the given continuous function, the fractal dimension is estimated. Further, the relation between its Hausdorff dimension and box dimension is explored.

Section 2 explores multifractal analysis on the Moran sets and its corresponding measures. For the relative multifractal formalism of non-regular Moran measures, some sufficient conditions are developed [22]. Further, the idea of a stochastic dyadic Cantor set is extended to a weighted planar stochastic lattice, which leads to a stochastic porous lattice [23].

The multifractal characterization of reference evapotranspiration in India, based on five different locations with different climatic conditions, is studied in [24]. Multifractal Cross Correlation Analysis is presented as a robust method for analyzing correlations between evapotranspiration and other predictor vari- ables like average wind speed, minimum and maximum air temperature.

In [25], a new fractal dimension, namely the $\phi$ topological Billingsley dimension, is presented, where $\phi$ is a non-negative function defined on the collection of subsets of separable metric space. The exact value of the $\phi$-topological Billingsley dimension for a familiar fractal set (the Sierpinski carpet) is estimated. In addition, the connections between the $\phi$-topological Billingsley dimension, Hausdorff dimension and topological Hausdorff dimension are presented together with an application.

In [26], multifractal analysis is used to characterize chaos in the finance system. The chaotic dynamics are analyzed for both noise-induced and noise-free systems. It is observed from multifractal analysis that noiseinduced systems manifest more complicated dynamics than the deterministic model.

In [27], a new approach to analyzing plasma dynamics via power spectral fractal analysis of the plasma emission spectrum is presented, taking copper plasma as an example. The correlation between the fractal dimension and the plasma temperature is revealed in order to develop a surrogate method for plasma temperature analysis.

In Sect. 3, the Hyers-Ulam stability for the fractional order fractal differential equations is investigated [28]. By taking time as a fractal set, the radioactive decay is mathematically modeled and it is shown that the model is Hyers-Ulam stable. Further, fractal calculus is applied to fractal Cantor cubes [29]. Fractal calculus is defined on the fractal cubes by making use of the integral staircase function. Moreover, the fractal Laplace equation is solved for the fractal sets and the Casimir effect is illustrated as its application.

Section 4 constructs a new kind of memristor by connecting a sine and cosine function memristor in series [30]. The dynamical properties, fractal dimension and Lyapunov exponents of the series memristor constructed are numerically investigated through its voltampere characteristics system. The circuit simulation results are compared with the numerical results and are found to match.

In [31], the construction of a $2 \mathrm{D}$ chaotic system is proposed and its chaotic characteristics are discussed. Multi-stability analysis is explored for different states of the chaotic system.

In [32], a general fractional calculus is described by using fractional operators with respect to another function. Together with the continuous time random walk, a generalized time-fractional Fokker-Planck equation is investigated.

In section 5, a mathematical model using fuzzy controllers is proposed for a two-link robot manipulator system [33]. Using random fractals, an optimal algorithm is presented to improve the proposed system's performance. To control the two-link robot manipulator, the fuzzy control system is evaluated in a simulated environment. The simulation results demonstrate that the proposed system provides better response curves in terms of stability and minimum settling time. 
In [34], the transmission of COVID-19 is analyzed using Generalized Fractal Dimensions. X-ray images from COVID-19 patients and healthy individuals are compared graphically. To determine the robustness of COVID-19 virus, multifractal dimension measure is evaluated for noisy, noise-free and original images.

In [35], a decision-making system using machinelearned classifiers and seven parameters to examine twenty-two crops is presented. Based on observations of the fractal dimension, it is found that the parameters follow anti-persistent behavior and are thus responsible for the maximal yield in smart farming. Of the three algorithms discussed, the random forest algorithm is the one that provides more accuracy in crop management systems. The study will help governments and farmers alike to make better decisions in crop management.

In [36], various types of topological index like the Randic index, and Harmonic index are estimated for two different fractal sets, namely the Sierpinski rhombus and Koch snowflake. Further, the calculated indices are compared with their respective fractal dimensions.

The editors of this special issue wish to thank the authors for their valued contributions, and the referees for their dedicated efforts in reviewing the articles. We believe that the selected papers gathered here will enrich readers' knowledge and will help scientists and researchers to further develop the theory of fractal analysis and related applications. Lastly, we wish to express our sincere gratitude to all members of EPJ ST for hosting this special issue.

\section{References}

1. M.F. Barnsley, Fractals Everywhere (Academic Press, Dublin, 2012)

2. P.R. Massopust, Fractal Functions, Fractal Surfaces and Wavelets (Academic Press, Dublin, 1994)

3. S. Banerjee, D. Easwaramoorthy, A. Gowrisankar, Fractal Functions, Dimensions and Signal Analysis (Springer, Cham, 2021)

4. S. Banerjee, M.K. Hassan, S. Mukherjee, A. Gowrisankar, Fractal Patterns in Nonlinear Dynamics and Applications (CRC Press, Boca Raton, 2020)

5. M.F. Barnsley, Constr. Approx. 2, 303-329 (1986)

6. M.A. Navascués, Acta Appl. Math. 110, 1199-1210 (2010)

7. S.K. Katiyar, A.K.B. Chand, M.A. Navascués, Rev. R. Acad. Cienc. Zaragoza 71, 7-30 (2016)

8. P. Grassberger, Phys. Lett. A 97, 227-320 (1983)

9. H.G.E. Hentschel, I. Procaccia, Phys. D 8(3), 435-444 (1983)

10. D. Easwaramoorthy, R. Uthayakumar, J. Comput. Sci. 2(1), 31-38 (2011)

11. R. Uthayakumar, D. Easwaramoorthy, Fluct. Noise Lett. 11(4), 1250034 (2012)

12. R. Uthayakumar, A. Gowrisankar, IET Image Process. 10(10), 751-762 (2016)

13. M.K. Hassan, Eur. Phys. J. Spec. Top. 228, 209-232 (2019)

14. T.M.C. Priyanka, A. Gowrisankar, Fractals 29(07), 2150215 (2021)
15. A. Gowrisankar, R. Uthayakumar, Mediterr. J. Math. 13(6), 3887-3906 (2016)

16. N. Vijender, V. Drakopoulos, Eur. Phys. J. Spec. Top. (2021). https://doi.org/10.1140/epjs/ s11734-021-00337-0

17. N. Akhtar, G.P. Prasad, N. Mohapatra, Eur. Phys. J. Spec. Top. (2021). https://doi.org/10.1140/epjs/ s11734-021-00321-8

18. N. Balasubramani, A. Gowrisankar, Eur. Phys. J. Spec. Top. (2021). https://doi.org/10.1140/epjs/ s11734-021-00306-7

19. V. Agrawal, T. Som, Eur. Phys. J. Spec. Top. (2021). https://doi.org/10.1140/epjs/s11734-021-00304-9

20. T.M.C. Priyanka, A. Gowrisankar, Eur. Phys. J. Spec. Top. (2021). https://doi.org/10.1140/epjs/ s11734-021-00315-6

21. M. Pandey, T. Som, S. Verma, Eur. Phys. J. Spec. Top. (2021). https://doi.org/10.1140/epjs/ s11734-021-00327-2

22. Z. Douzi, B. Selmi, A.B. Mabrouk, Eur. Phys. J. Spec. Top. (2021). https://doi.org/10.1140/epjs/ s11734-021-00318-3

23. T. Mitra, M.K. Hassan, Eur. Phys. J. Spec. Top. (2021). https://doi.org/10.1140/epjs/s11734-021-00329-0

24. S. Adarsh, L.J. Nityanjaly, Q.B. Pham, R. Sarang, M. Ali, P. Nandhineekrishna, Eur. Phys. J. Spec. Top. (2021). https://doi.org/10.1140/epjs/ s11734-021-00325-4

25. A.B. Mabrouk, B. Selmi, Eur. Phys. J. Spec. Top. (2021). https://doi.org/10.1140/epjs/ s11734-021-00313-8

26. S. Mukherjee, N.A.A. Fataf, M.F.A. Rahim, H. Natiq, Eur. Phys. J. Spec. Top. (2021). https://doi.org/10. 1140/epjs/s11734-021-00305-8

27. S. Sankararaman, Eur. Phys. J. Spec. Top. (2021). https://doi.org/10.1140/epjs/s11734-021-00328-1

28. A.K. Golmankhaneh, C. Tunç, H. Şevli, Eur. Phys. J. Spec. Top. (2021). https://doi.org/10.1140/epjs/ s11734-021-00316-5

29. A.K. Golmankhaneh, S.M. Nia, Eur. Phys. J. Spec. Top. (2021). https://doi.org/10.1140/epjs/ s11734-021-00317-4

30. J. Wang, Y. Cao, J. Mou, X. Liu, Eur. Phys. J. Spec. Top. (2021). https://doi.org/10.1140/epjs/ s11734-021-00330-7

31. X. Han, J. Mou, T. Liu, Y. Cao, Eur. Phys. J. Spec. Top. (2021). https://doi.org/10.1140/epjs/ s11734-021-00331-6

32. F. Hui, W. Guo-Cheng, G. Yang, L.-L. Huang, Eur. Phys. J. Spec. Top. (2021). https://doi.org/10.1140/ epjs/s11734-021-00323-6

33. T.A. Mai, T.S. Dang, Eur. Phys. J. Spec. Top. (2021). https://doi.org/10.1140/epjs/s11734-021-00339-y

34. Q. Rong, C. Thangaraj, D. Easwaramoorthy, S. He, Eur. Phys. J. Spec. Top. (2021). https://doi.org/10.1140/ epjs/s11734-021-00336-1

35. R. Bhardwaj, S. Bhardwaj, M. Sajid, B. Selmi, Eur. Phys. J. Spec. Top. (2021). https://doi.org/10.1140/ epjs/s11734-021-00333-4

36. A. Divya, A. Manimaran, Eur. Phys. J. Spec. Top. (2021). https://doi.org/10.1140/epjs/ s11734-021-00338-z 